\title{
Higher Spectral Efficiency Coherent Optical OFDM Transmission With Iterative Polar Modulation
}

\author{
K.Sharmila Kumari ${ }^{1}$, M.Ashok Kumar ${ }^{2}$ \\ ${ }^{1}(P G$ Scholar in Communication Systems, Adhiyamaan College Of Engineering, Hosur, India) \\ ${ }^{2}$ (AP in Electronics and Communication Engineering, Adhiyamaan College Of Engineering, Hosur, India)
}

\begin{abstract}
Achieving high spectral efficiency in optical transmissions has recently attracted much attention, aiming to satisfy the ever increasing demand for high data rates in optical fiber communications. Therefore, strong Forward Error Correction (FEC) coding in combination with multilevel modulation schemes is mandatory to approach the channel capacity of the transmission link. In this paper we give design rules on the joint optimization of coding and signal constellations. We propose a novel coded modulation scheme based on 512-IPM for a fiber optical transmission link of $800 \mathrm{~km}$. The spectral efficiency achieved was $10 \%$ greater i.e, $12.1 \mathrm{~b} / \mathrm{s} / \mathrm{Hz}$ at a post FEC BER of $<10^{-15}$ by using coded optimized constellation. In detail, the "Turbo Principle" to BICM-ID is adapted and combine it with a high-rate outer algebraic code to obtain a post-FEC $B E R<10^{-15}$, which is a typical demand in optical transponders. Furthermore we give simple design principles for the design of BICM-ID based on the extrinsic information transfer (EXIT) chart analysis.

Keywords: Bit interleaved coded modulation with iterative decoding (BICM-ID), Iterative-Polar-Modulation (IPM), Coherent optical orthogonal frequency division multiplexing (CO-OFDM), Spectral efficiency.
\end{abstract}

\section{Introduction}

Over the last decade there has been an exponential growth of bandwidth-intensive services such as video on demand, cloud storage and social networking which require large volumes of data to be transmitted over long distances. Based on current trends, this growth is likewise to continue, driving the need to increase transmission capacity. Ongoing commercial developments with data rates of $100 \mathrm{Gbit} / \mathrm{s}$ in the standardized 50 $\mathrm{GHz}$ wavelength-division multiplexing (WDM) grid consider polarization division multiplexed quadrature phase shift keying (PDM-QPSK) as the most suitable modulation format [1]. For channel data rates beyond 100$\mathrm{Gb} / \mathrm{s}$, e.g., 400-Gb/s, exploring modulation formats offering higher spectral efficiency (SE) than PDM-QPSK becomes necessary. The most effective method to increase the spectral efficiency of any communication system is to employ larger modulation formats [2].

Recently, using Nyquist-pulse-shaped PDM-32-point quadrature amplitude modulation (QAM) [3] and 64-QAM [4], WDM transmission over 400-km with $8.37-\mathrm{b} / \mathrm{s} / \mathrm{Hz}$ SE and over $240-\mathrm{km}$ with $10-\mathrm{b} / \mathrm{s} / \mathrm{Hz}$ SE has been demonstrated. Using CO-OFDM [5] with PDM-128-QAM subcarrier modulation, WDM transmission with $\mathrm{SE}$ and $165-\mathrm{km}(3 \times 55-\mathrm{km})$ reach has been reported [6]. For single-channel transmission, Nyquist-pulse-shaped PDM-256-QAM [7], PDM-512-QAM [8] and OFDM-PDM-1024-QAM [9] have been used to achieve remarkable ISEs of $16-\mathrm{b} / \mathrm{s} / \mathrm{Hz}, 12.4-\mathrm{b} / \mathrm{s} / \mathrm{Hz}$ and $11.7-\mathrm{b} / \mathrm{s} / \mathrm{Hz}$, respectively, but the achieved transmission reach was limited to $160-\mathrm{km}$, and the net channel data rates were less than $64-\mathrm{Gb} / \mathrm{s}$. To increase the transmission reach and data rate of high-SE signals, forward error correction (FEC) coding schemes with soft-decision (SD) decoding is being actively studied [10]. Coded iterative polar modulation (IPM) was recently studied numerically to achieve higher coding gain than conventional SD-FEC schemes in high-SE optical transmission [2].

The remainder of the paper is organized as follows: In Section II the design principles of capacity achieving constellations are derived. In Section III, the investigated optical OFDM system is introduced. The basic coded modulation scheme, namely BICM-ID, is reviewed in Section IV. Design principles for BICM-ID with IPQ signal constellations are given in Sections V. We conclude our paper in Section VII.

\section{Design Of Capacity Approaching Constellations By Means Of Iterative Polar Quantization}

A more efficient shaping method based on optimized nonuniform signal constellations for providing a quasi-Gaussian source is considered. Design principles of capacity achieving constellations are proposed in [11]. Shaped signal constellation is designed by using 512-iterative polar quantization. In order to provide Gaussian signalling at the input of the system, optimized the signal constellation $A=\left\{a_{0}, a_{1}, \ldots a_{Q-1}\right\}$ by minimizing the quantization mean square error (QMSE) of a Gaussian information source by means of iterative polar quantization (IPQ). The constellation points of the obtained set are distributed over circles of radii determined by a Rayleigh distribution. Using a Rayleigh distribution $\mathrm{p}(\mathrm{r})$ originates from the fact that the envelope $\mathrm{r}$ of a 
two-dimensional Gaussian distribution is Rayleigh distributed. Let $\mathrm{N}_{\mathrm{r}}$ denote the number of circles in the constellation and $L_{j}$ denote the number of constellation points per circle of radius $m_{j} ; j \in\{1,2, \ldots, N r\}$. Then the optimum number of constellation points at the $\mathrm{j}$-th circle for a Q-ary signal set is determined by minimizing the QMSE of a Gaussian source

$L_{j}=\sqrt[3]{m_{j}^{2} \int_{r_{j}}^{r_{j+1}} p(r) d r} /\left[\sum_{j=2}^{N r} \frac{1}{2^{M}} \sqrt[3]{m_{j}^{2} \int_{r_{j}}^{r_{j+1}} p(r) d r}\right]$

The radius of the $j$-th circle is calculated as

$m_{j}=\left[2 \sin \left(\frac{\Delta \theta_{j}}{2}\right)\right] \int_{r_{j}}^{r_{j+1}} p(r) d r /\left[\Delta \theta_{j} \int_{r_{j}}^{r_{j+1}} p(r) d r\right]$

Where $\Delta \Theta_{\mathrm{j}}=2 \pi / \mathrm{k}_{\mathrm{j}}$. The limits of integration in (1) and (2) are determined by

$r_{j}=\frac{\pi\left(m_{j}^{2}-m_{j-1}^{2}\right) / 2}{m_{j} k_{j} \sin \left(\frac{\Delta \theta_{j}}{2}\right)-m_{j-1} k_{j-1} \sin \left(\frac{\Delta \theta_{j-1}}{2}\right)}$

The optimum signal constellation for a Q-ary signal set is found by applying (1)-(3) in an iterative fashion until convergence is reached. Referring square constellations to Q-ary QAM, the optimized constellations based on IPQ are called Q-ary IPM. All IPM constellations investigated here were designed for a Gaussian source with variance $\sigma^{2}=1$. The 512-IPM constellation obtained through the above procedure is applied in the experimental study presented in the following sections of this paper. The distribution of the constellation points is nonuniform and follows a discrete 2D Gaussian distribution with zero mean. Table 1 summarizes the details of the 512-IPM constellation

To assess the benefit of utilizing IPM signal constellations instead of square QAM, calculated the capacity of the signal constellation A per unit bandwidth as

$c(A)=1 / Q \sum_{a \in A} \operatorname{fr}\left(\frac{r}{a}\right) \log 2 \frac{\operatorname{fr}\left(\frac{r}{a}\right)}{\sum_{a \in A} \operatorname{fr}\left(\frac{r}{a}\right)} d r$

It is assumed that equal a-priori probability for the input symbols $a_{m} \in A, p\left(a_{m}\right)=1 / Q$ and use the conditional probability density function (PDF) of the complex valued AWGN channel

$\begin{aligned} \operatorname{fr}\left(\frac{\mathrm{r}}{\mathrm{a}}\right) & =1 / 2 \pi \sigma 2 \exp \left(-\frac{1}{2 \sigma 2}|\mathrm{r}-\mathrm{a}| 2\right) \\ & =\mathrm{N}(\mathrm{a}, \sigma 2)\end{aligned}$

where the variance $\sigma^{2}$ corresponds to the double-sided noise power spectral density $N_{0} / 2$.

\begin{tabular}{|c|c|c|c|}
\hline \multicolumn{5}{|c|}{ Table.1 summary of the specifications for 512 IPM } \\
\hline $\mathrm{N}_{\mathrm{r}}$ & $\mathrm{r}_{\mathrm{j}}$ & $\mathrm{m}_{\mathrm{j}}$ & $\mathrm{L}_{\mathrm{j}}$ \\
\hline 1 & 0.00 & 0.11 & 4 \\
\hline 2 & 0.18 & 0.27 & 11 \\
\hline 3 & 0.35 & 0.43 & 23 \\
\hline 4 & 0.51 & 0.59 & 29 \\
\hline 5 & 0.67 & 0.76 & 34 \\
\hline 6 & 0.84 & 0.93 & 39 \\
\hline 7 & 1.02 & 1.11 & 43 \\
\hline 8 & 1.20 & 1.30 & 47 \\
\hline 9 & 1.40 & 1.50 & 49 \\
\hline 10 & 1.61 & 1.72 & 50 \\
\hline 11 & 1.84 & 1.96 & 46 \\
\hline 12 & 2.09 & 2.23 & 41 \\
\hline 13 & 2.39 & 2.55 & 30 \\
\hline 14 & 2.75 & 2.95 & \\
\hline 15 & 3.25 & 3.52 & \\
\hline 16 & 4.50 & & \\
\hline
\end{tabular}




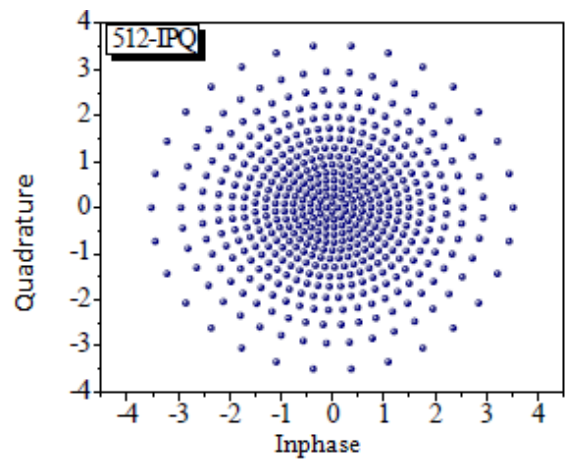

Fig.1. The 512 IPM signal constellation

III. The Coherent Optical OFDM System

The goal is to enable high speed communication $\left(100^{+}-\mathrm{Gb} / \mathrm{s}\right)$ at high spectral efficiency, while being robust against frequency offset between the OLO and the transmit laser in the presence of large fiber dispersion. Orthogonal frequency division multiplexing (OFDM) belongs to multicarrier transmission, where a single data stream is transmitted over a number of lower rate orthogonal subcarriers. CO-OFDM combines the advantages of 'coherent detection' and 'OFDM modulation' and has many merits that are critical for future high-speed fiber transmission systems.

\subsection{Principle of orthogonal frequency division multiplexing}

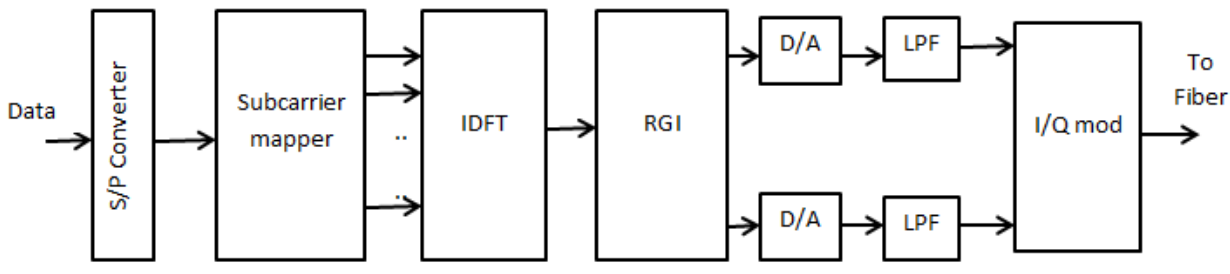

(a)

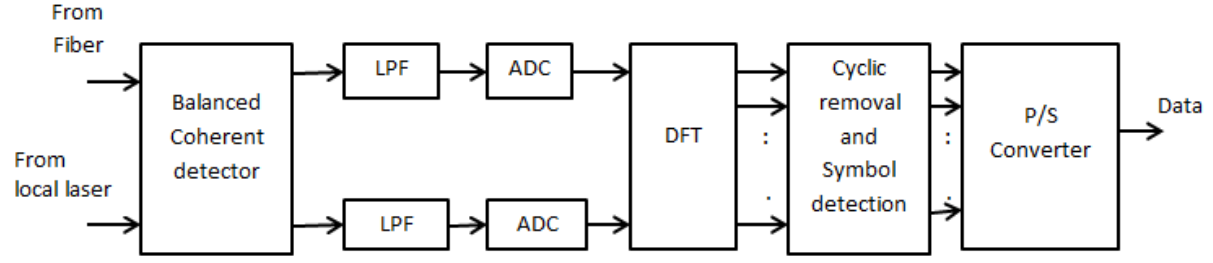

(b)

Fig.2. The CO-OFDM system architecture (a) CO-OFDM transmitter configuration (b) COOFDM receiver configuration

A fundamental challenge with the OFDM is that a large number of subcarriers are needed so that the transmission channel affects each subcarrier as a flat channel. This leads to an extremely complex architecture involving many oscillators and filters at both transmit and receive end. Weinsten and Ebert first revealed that OFDM modulation/demodulation can be implemented by using Inverse Discrete Fourier Transform (IDFT)/Discrete Fourier Transform (DFT) [5]. The modulation can be performed by IDFT of the input information symbol and the demodulation by DFT of the sampled received signal. The corresponding architecture using DFT/IDFT and digital-to-analog/analog-to-digital converter (DAC/ADC) are shown in Fig. 2. At the transmit end, the input data bits are mapped onto corresponding information symbols of the subcarriers within one OFDM symbol, and the digital time domain signal is obtained by using IDFT, which is subsequently inserted with guard interval and converted into real time waveform through DAC. The guard interval is inserted to prevent inter-symbol-interference (ISI) due to channel dispersion. The baseband signal can be up-converted to an appropriate RF band with an IQ mixer/modulator. In setup, the IDFT size was set to 128, and the GI includes 2 samples, resulting in a small GI-overhead of $1.56 \%$. The null-to-null spectral bandwidth of the RGI-COOFDM signal was $20.75 \mathrm{GHz}$. At the receive end, the OFDM signal is first down-converted to baseband with an 
IQ demodulator, and sampled with an ADC, and demodulated by performing DFT and baseband signal processing to recover the data.

\subsection{Cyclic prefix for OFDM}

Cyclic prefix was proposed to resolve the channel dispersion induced ISI and ICI [12].In this paper the RGI-CO-OFDM scheme is applied. In RGI-CO-OFDM, a reduced GI or CP between adjacent OFDM symbols is used to accommodate the ISI with short memory, such as induced by transmitter and receiver band width limitations or fiber PMD, while fiber CD-induced ISI with long memory and well-defined characteristics is compensated at the receiver, like in single-carrier systems. In essence, RGI-CO-OFDM is a hybrid version of conventional CO-OFDM with $\mathrm{CP}$ and single-carrier frequency-domain equalization (SC-FDE).

\section{Spectral Efficient Coding In Optical Communications}

A FEC scheme relying on the concatenation of a low-redundancy algebraic outer Reed-Solomon (RS) code and an inner coded modulation scheme, based on the concept of bit-interleaved coded modulation with iterative decoding (BICM-ID) is proposed. The FEC scheme is depicted in Fig. 3.

Outer algebraic coding is mandatory to obtain very low BER, since the inner scheme relies on the "Turbo Principle" and may run into an error floor which is several decades higher than the demanded post-FEC BER of $<10^{-15}$. More specifically, considered the RS $(2720,2550)$ code over Galois-field GF (12), with a code rate of $R_{R S}=0.9375$ and a code word $(C W)$ length of 32640 for the outer coding. This particular code supplies a net coding gain (NCG) of $8-\mathrm{dB}$ at a pre-FEC BER of $1.1 \times 10^{-3}[30]$.

For inner coding, the "Turbo Principle" to BICM-ID, using soft-decision decoding is adapted[13]. In contrast to the original proposal on BICM-ID, the low-density parity-check (LDPC) component codes are used. The benefit of using LDPC codes compared to convolutional codes is that these codes are easy to implement and we can omit interleaves by using a special check matrix decomposition for encoding, which also leads to a fairly random distribution of individual bit positions in a $\mathrm{CW}$. Since we used binary codes, the modulation size $\mathrm{Q}$ needs to be a potency of $2\left(\mathrm{Q}=2^{\mathrm{M}}\right)$. Therefore, after binary encoding, the grouping operation took $\mathrm{M}$ coded bits $\mathrm{x}_{0,1 \ldots \mathrm{M}-1} \epsilon\{0,1\}$ to form the complex output symbol $\mathrm{a}=\mu\left(\mathrm{t}_{0,1, \ldots, \mathrm{M}-1}\right)$. This mapping operation $\mu(\cdot)$ is essential for the iterative demapping and decoding process, since it links up the bits $\mathrm{x}_{0,1 \ldots \mathrm{M}-1}$ to a symbol and mutual dependencies arise between these bits.

The encoded symbols were then fed to the CO-OFDM system and transmitted over the channel. Due to the use of OFDM, the central limit theorem applies when performing the DFT on the received channel symbols, leading to the fact that after equalization the symbols $r$ at the input of the decoder were corrupted by fairly Gaussian distributed noise $\mathrm{r}=\mathrm{a}+\mathrm{n}$ only, where $\mathrm{n}$ is a complex Gaussian random variable with variance $\sigma^{2}$.

In the decoder, the soft-demapper calculates an a posteriori probability (APP) soft-output loglikelihood ratio (LLR) [13]

$$
L_{p}^{\mu}\left(x_{k}\right)=\ln \frac{p\left(x_{k}=1 \mid r\right)}{p\left(x_{k}=0 \mid r\right)}
$$

For each of the M coded bits per symbol,

$L_{p}^{\mu}\left(x_{k} / r\right)=L_{a}^{\mu}\left(x_{k}\right)+\ln \frac{\sum_{a \in A_{k}^{1}} \exp \left[-\frac{|r-a|}{2 \sigma^{2}}+\sum_{j=1, j \neq k}^{M} \mu_{j}^{-1}(a) L_{a}^{\mu}\left(x_{k}\right)\right]}{\sum_{a \in A_{k}^{0}} \exp \left[-\frac{|r-a|}{2 \sigma^{2}}+\sum_{j=1, j \neq k}^{M} \mu_{j}^{-1}(a) L_{a}^{\mu}\left(x_{k}\right)\right]}$

where $L_{a}{ }^{\mu} x(k)$ is the a priori soft-input LLR of bit $x_{k}$ being rather 1 or $0, \mu_{j}{ }^{-1}\left(a_{m}\right)$ gives the $j$-th bit of the binary decomposition of symbol $\mathrm{a}_{\mathrm{m}}$ and $\mathrm{A}_{\mathrm{k}}{ }^{\mathrm{P}}, \mathrm{A}_{\mathrm{k}}{ }^{0}$ are the sets of symbols having the $\mathrm{k}$-th bit set to 1 and 0 ,respectively.

For the iterative demapping and decoding it is necessary to exchange only extrinsic information. Therefore the extrinsic information at the decoder output is the difference of the soft-input soft-output LLRs, $\mathrm{L}_{\mathrm{e}}{ }^{c}=\mathrm{L}_{\mathrm{p}}{ }^{\mathrm{c}}-\mathrm{L}_{\mathrm{e}}{ }^{\mu}$ which is fed back as a priori information of the soft-demapper $\mathrm{L}_{\mathrm{a}}{ }^{\mu}=\mathrm{L}_{\mathrm{e}}{ }^{\mathrm{c}}$. The demapper uses the extrinsic information from the decoder to calculate improved a posteriori LLRs $\mathrm{L}_{\mathrm{p}}{ }^{\mu}$. The extrinsic fraction of the a posteriori soft-output LLRs $\mathrm{L}_{\mathrm{p}}{ }^{\mu}$, so the difference of a priori and a posterior LLR $\mathrm{L}_{\mathrm{e}}{ }^{\mu}=\mathrm{L}_{\mathrm{p}}{ }^{\mu}-\mathrm{L}_{\mathrm{e}}{ }^{\mathrm{c}}$ is passed to the LDPC decoder for further iterative decoding steps. The iterative demapping and decoding process was stopped when the LDPC decoder determined a valid CW or a maximum number of 13 iterations was reached. Further, the samples at the input of the soft-demapper $r$ had a resolution of 8 bit and the LLRs were represented with 6 bit. 


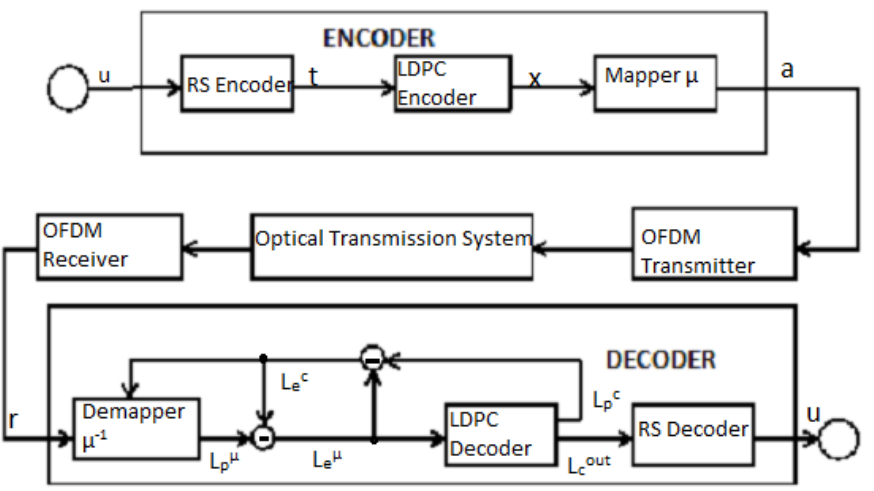

Fig.3. Concatenated iterative soft demapping and decoding scheme.

\section{Design Of BICM-ID For IPM}

As noted above, residual errors were removed by the outer RS $(2720,2550)$ code. Therefore, the inner coded modulation scheme needs to acquire a post-FEC BER below the threshold BER of the outer RS code of $1.1 \times 10^{-3}$, in order to obtain a total post-FEC $<10^{-15}$. The joint design of mapping and coding was performed by using the EXIT chart analysis, which is a powerful tool to visualize the flow of extrinsic information between the soft-demapper and decoder [14].

The idea of the EXIT chart analysis is to predict the behavior of the iterative decoding loop by measuring the relation between a priori $M I I_{a}$ and extrinsic $M I I_{e} \cdot X_{a}$ and $X_{e}$ as two real valued random variables, then we can compute the mutual information (MI) $\mathrm{I}\left(\mathrm{x}_{\mathrm{e}} ; \mathrm{x}_{\mathrm{a}}\right)$ by

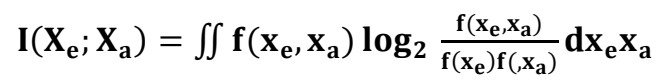

For the simulation of the iterative decoding loop assumed that the a prior soft-input LLRs $\mathrm{L}_{\mathrm{a}}$ and the extrinsic soft-output LLR $\mathrm{L}_{\mathrm{e}}$ are fairly uncorrelated from the respective channel observations over many iterations and are Gaussian-like distributed due to the fact that central limit theorem applies in iterative decoding [18]. For the design of the EXIT function of the soft-demapper we needed to measure the MI between the bits $\mathrm{x}$ which were after the mapping transmitted over a noise corrupted channel and the soft-output LLR of these bits after soft-demapping. In this simulation, considered an AWGN channel denoted by $\mathrm{N}\left(0, \sigma_{\mathrm{ch}}{ }^{2}\right)$ The soft-demapper received the channel symbols $r$ and soft-input a priori knowledge as LLRs $L_{a}$ of the decoder, which were modelled by the output of a virtual channel denoted by $\mathrm{N}\left(0, \sigma_{\mathrm{a}}{ }^{2}\right)$ normalized by the channel state information $2 /$ $\sigma_{\mathrm{a}}{ }^{2}$. In order to obtain the relation between a priori MI $\mathrm{I}_{\mathrm{a}}$ and extrinsic MI $\mathrm{I}_{\mathrm{e}}$ we needed measure the MI between the bits $\mathrm{x}$ and the output of the virtual channel $\mathrm{I}\left(\mathrm{L}_{\mathrm{a}}, \mathrm{X}\right)$ and the extrinsic output of the soft-demapper $\mathrm{I}\left(\mathrm{L}_{\mathrm{e}}, \mathrm{X}\right)$, respectively. By invoking the ergodic theorem we performed this measurement for a large number of samples by

$\mathrm{I}(\mathrm{L}, \mathrm{X})=1-\mathrm{E}\left[\log _{2}\left(1+\mathrm{e}^{-\mathrm{L}}\right)\right] \log _{2}\left(1+\mathrm{e}^{-\mathrm{xnLn}}\right)$

$\approx 1-\frac{1}{N} \sum_{n-1}^{N} \log _{2}\left(1+e^{-x_{n} L_{n}}\right)$

Fig. 4 shows the model for the measurement of the EXIT function of the soft-demapper. The measurement of the LDPC decoder EXIT function was similar, but with the difference that the LDPC decoder only obtained a priori knowledge, since it is not directly attached to the channel.

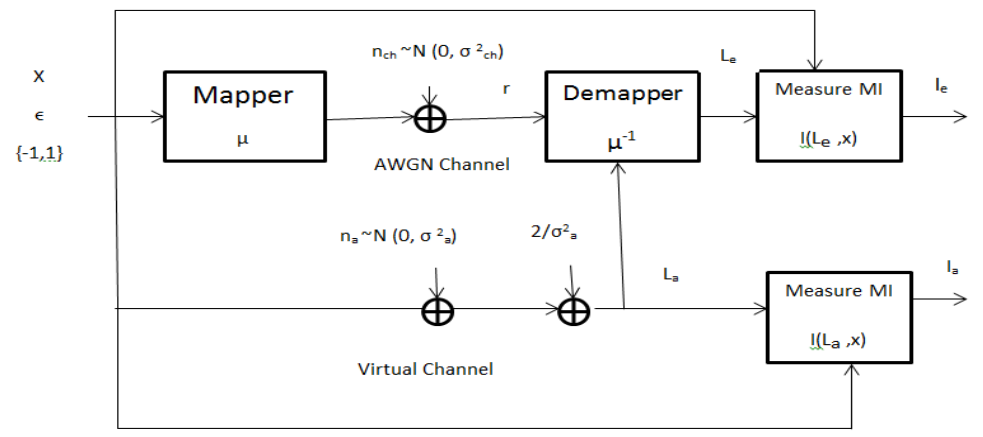

Fig.4. Measurement of the soft-demapper EXIT function 
In our proposed coded modulation scheme the mapping of the modulation was Gray-like for 512-IPM to reduce the number of iterations between the soft-demapper and decoder. A pure Gray mapping for IPM was not available due to the non-uniform circular distribution of the signal constellation points. On the basis of the EXIT-function of the soft-demapper, a proper degree distribution for an LDPC code was found by means of differential evolution. For both modulations, the code rate was fixed to 0.778 , resulting in a total FEC overhead of $37.1 \%$. Furthermore, the CW length of the LDPC code was 40032 and a maximum of 20 LDPC iterations were performed. Figure 5 shows the EXIT chart of the designed coding scheme. Obviously, the EXIT functions of the soft-demapper and decoder intersect at an MI corresponding to a BER $<1.1 \times 10^{-3}$ without performing any iterations between the decoder and demapper.

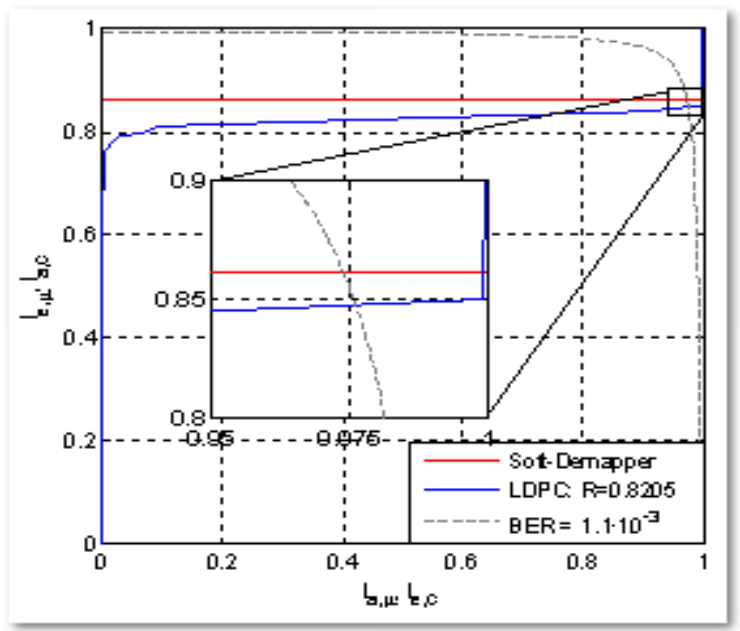

Fig.5. EXIT chart for the designed signal constellation

\section{Result And Discussion}

Figure 6 shows the BER performance of multiplexed 512 IPM transmission. Observations had been done based on the change of sampling signals. It can be seen that a post-FEC BER $<10^{-15}$ is achieved.
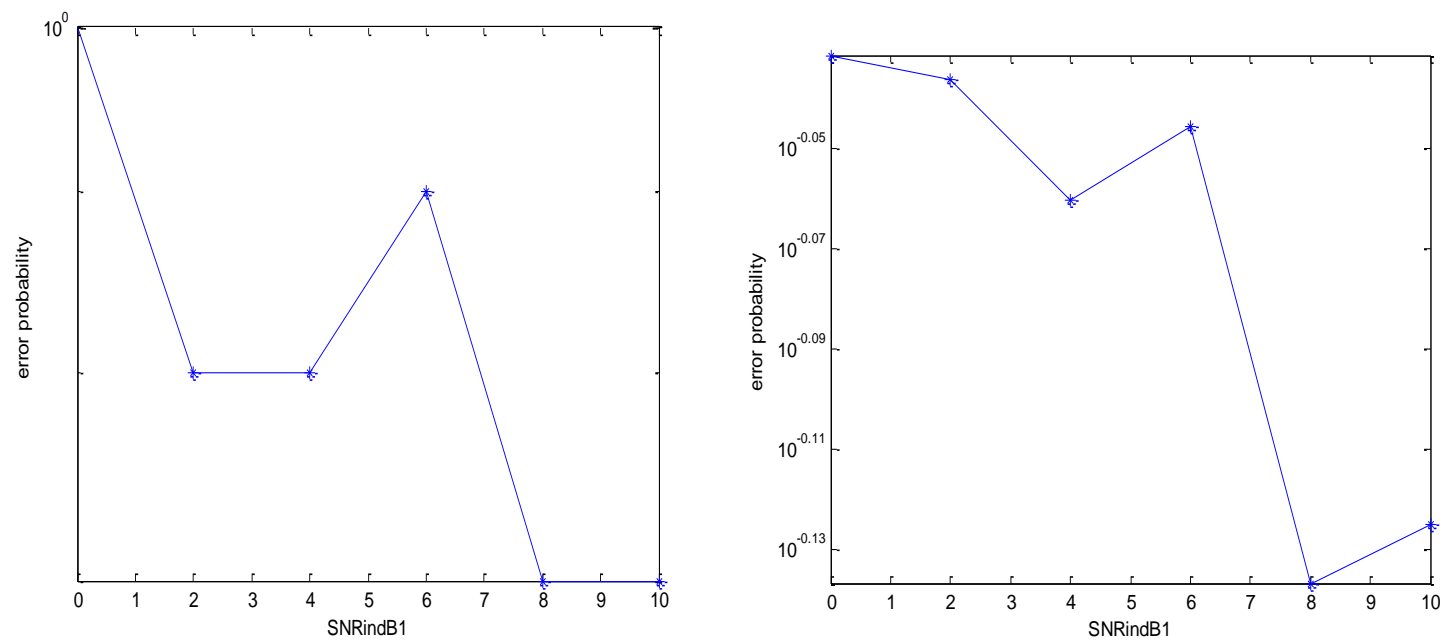

(a) (b) 


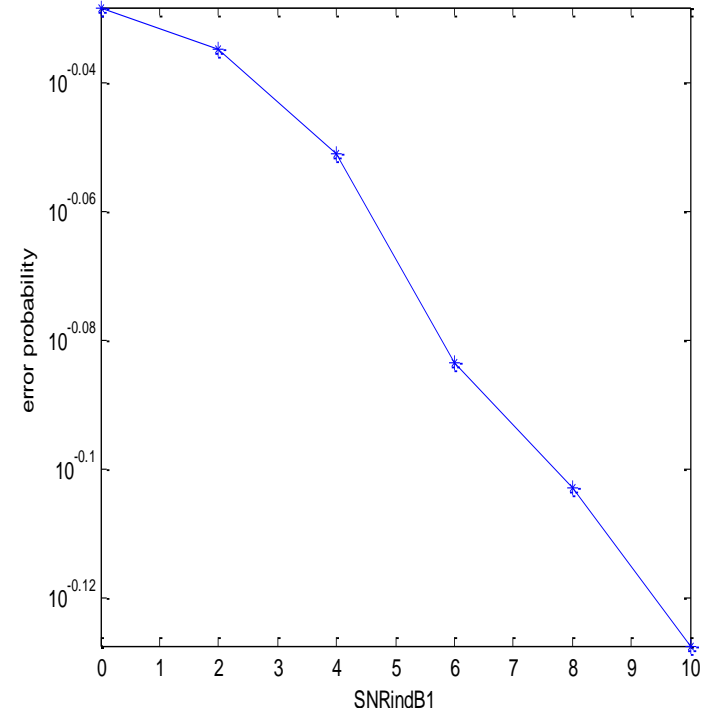

(c)

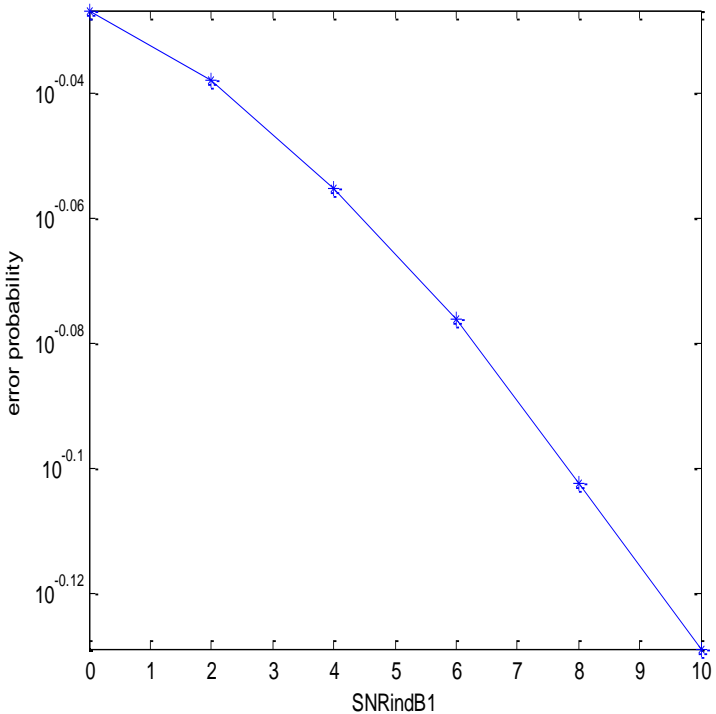

(d)

Fig.6. BER performance for (a) $n=10$ (b) $n=100$ (c) $n=1000$ and (d) $n=10000$ samples

\section{Conclusion}

In this paper we presented a simple, but spectral highly efficient coding scheme for optical OFDMbased communications, relying on the concatenation of BICM-ID with a high-rate outer code to obtain a postFEC BER $<10^{-15}$. Further, we provided design principles of capacity approaching constellations and showed how to jointly optimize mapping and coding for a proper convergence of the iteration loop. The net spectral efficiency achieved is $12.1 / \mathrm{b} / \mathrm{s} / \mathrm{Hz}$ the parameter considered here to show spectral efficiency is BER. The spectral efficiency achieved can support high speed transmission over long distances. This study shows the promise of combining coding with modulation (e.g., constellation shaping) and nonlinear compensation to achieve a substantially higher optical transmission performance than using coding alone.

\section{References}

[1] R.Nagarajan, D.Lambert, M.Kato, V.Lal, G.Goldfarb, J.Rahn, et.aL,'10 Channel 100Gbit/s per Channel, Dual Polarisation, Coherent QPSK, Monolithic Inp Receiver Photonic Integrated Circuit', in Proc. OFC/NFOEC, OML7, San Diego, CA, USA, 2011.

[2] T. Lotz, W. Sauer-Greff, and R. Urbansky, "Capacity approaching coded modulation in optical communications," in Proc. ICTON'11,Tu.D1.2.

[3] X. Zhou, L. E. Nelson, P. Magill, B. Zhu, and D. W. Peckham, " $8 \mathrm{X} 450-\mathrm{Gb} / \mathrm{s}, 50-\mathrm{GHz}-\mathrm{spaced}$, PDM-32 QAM transmission over400-km and one $50 \mathrm{GHz}$-hrid ROADM," in Proc. OFC'11, Post-Deadline Paper PDPB3.

[4] T. Kobayashi, A. Sano, A. Matsuura, M. Yoshida, T. Sakano, H.Kubota, Y. Miyamoto, K. Ishihara, M. Mizoguchi, and M. Nagatani,"45.2 Tb/s C-band WDM transmission over 240-km using 538-Gb/s PDM-64 QAM single carrier FDM signal with digital pilot tone,"in Proc. ECOC'11, Post-Deadline Paper Th.13.C.6.

[5] W. Shieh, H. Bao, and Y. Tang, "Coherent optical OFDM: Theory and design," Opt. Exp., vol. 16, pp. 841-859, 2008.

[6] D. Qian, M.-F. Huang, E. Ip, Y.-K. Huang, Y. Shao, J. Hu, and T. Wang, "101.7-Tb/s (370 X294-Gb/s) PDM-128 QAM-OFDM transmission over 3X 55-km SSMF using pilot-based phase noise mitigation,"in Proc. OFC'11, Post-Deadline Paper PDPB5.

[7] R. Schmogrow, D. Hillerkuss, S. Wolf, B. Bäuerle, M. Winter, P. Kleinow, B. Nebendahl, T. Dippon, P. C. Schindler, C. Koos, W. Freude, and J. Leuthold, "512 QAM Nyquist sinc-pulse transmission at 54 Gbit/s in an optical bandwidth of $3 \mathrm{GHz}$,"Opt. Exp., vol. 20, pp. 6439-6447, 2012.

[8] S. Okamoto, K. Toyoda, T. Omiya, K. Kasai, M. Yoshida, and M. Nakazawa, "512 QAM (54 Gbit/s) coherent optical transmission over150-km with an optical bandwidth of $4.1 \mathrm{GHz}$," in Proc. ECOC' 10, Post-Deadline Paper PD.2.3.

[9] M.-F. Huang, D. Qian, and E. Ip, "50.53-Gb/s PDM-1024QAMOFDM transmission using pilot-based phase noise mitigation," in Proc. OECC' 11, pp. 752-753.

[10] F. Chang, K. Onohara, and T. Mizuochi, "Forward error correction for $100 \mathrm{G}$ transport networks," IEEE Commun. Mag. , vol. 48, pp. 48-55, 2010.

[11] Z. H. Peric, I. B. Djordjevic, S. M. Bogosavljevic, and M. C. Stefanovic, "Design of signal constellations for Gaussian channel by Iterative polar quantization," in Proc. 9th Mediterranean Electrotech. Conf., May 1998, vol. 2, pp. 866-869.

[12] J. Armstrong, "OFDM for optical communications," J. Lightw. Technol., vol. 27, pp. 189-204, 2009.

[13] S. ten Brink, J. Speidel, and R.-H. Yan, "Iterative demapping and decoding for multilevel modulation," in Proc. IEEE GLOBECOM, 1998, pp. 579-584.

[14] S. ten Brink, "Convergence behavior of iteratively decoded parallel concatenated codes," IEEE Trans. Commun., vol. 49, no. 10, pp.1727-1737, 2001. 